\title{
Effects of cell motility and morphology on the rheology of algae suspensions
}

\author{
N. Cagney • T. Zhang • R. Bransgrove • \\ M. J. Allen • S. Balabani
}

6 Received: date / Accepted: date

\begin{abstract}
Algae have been proposed as a source of biofuels and high value chemical products, but if this potential is to be fully realised, it is crucial to understand the factors affecting the suspension rheology. Suspensions of three algae species, Tetraselmis chuii, Chlorella sp. and Phaeodactylum tricornutum, were sheared in a rotational rheometer in order to characterise their rheology and examine the effects of cell concentration, motility and morphology. The volume fraction ranged from 0.05 to 0.2 , and the shear rate from 20 to $200 \mathrm{~s}^{-1}$. The rheology measurements are fitted to the Herschel-Bulkley model, and the intrinsic viscosity is estimated using both Einstein's equation and the KriegerDougherty model, which are found to perform well for low concentrations. The intrinsic viscosity of $T$. chuii suspensions is shown not to be constant, but decreases with strain rate, indicating that the suspension viscosity is less sensitive to the cell concentration at high strain rates. The rate of decline is constant for strain rates below approximately $100 \mathrm{~s}^{-1}$, after which it continues to decline linearly but at a slower rate. It is speculated that this transition at
\end{abstract}

\section{N. Cagney}

Department of Earth Sciences, University College London

\section{T. Zhang}

Department of Mechanical Engineering, University College London

R. Bransgrove

Plymouth Marine Laboratory

M. J. Allen

Plymouth Marine Laboratory

S. Balabani

Department of Mechanical Engineering, University College London

E-mail: s.balabani@ucl.ac.uk 
$100 \mathrm{~s}^{-1}$ is related to the appearance of flocculation at low strain rates. The effect of the cell motility on the rheology of T. chuii suspensions is investigated by comparing the rheology of motile and passive cells. The shear-thinning behaviour is absent and the effective viscosity is considerably lower for the passive cell suspensions, indicating that the motility of the T. chuii cells causes them to align to resist the flow. In contrast, the $C$. $s p$. suspensions exhibit shear-thickening behaviour, which has not previously been reported. Finally, the influence of the effective aspect ratio on the cell suspensions is examined by comparing the intrinsic viscosity of all three species. The algal species with the largest aspect ratio, $P$. tricornutum, has the largest intrinsic viscosity, while the smallest aspect ratio strain, $C . s p$., has the smallest viscosity. However, it is shown that the increase in viscosity of motile compared to non-motile $T$. chuii suspensions cannot be attributed to a change in the effective aspect ratio of individual cells due to the motion of the flagella alone.

Keywords Algae suspension · effective viscosity · non-Newtonian · cell motility $\cdot$ cell morphology

\section{Introduction}

Increased levels of energy demand over the past century and the global reliance on fossil fuels have led to staggering levels of $\mathrm{CO}_{2}$. There is an urgent need to develop sustainable energy technologies, and microalgae have long been mooted as a potential solution. The potential of algae as an energy source is further increased by the possibility of using genetically modified species with higher lipid content (Radakovits et al, 2010). As microalgae are responsible for up to half of the carbon fixation on the Earth (Fields et al, 1998), they also present the possibility for effective carbon capture schemes to combat climate change. However, in spite of the theoretical potential of microalgae as a global energy source, many major challenges remain, including the efficiency of conversion of solar energy into fuels compared to other crops (Walker, 2009) and their requirement for limited resources, such as phosphorous and freshwater (Borowitzka and Moheimani, 2013). As a result, the commercial use of microalgae has tended to be limited to producing high-value chemicals for niche markets (Borowitzka, 2013), for which the economies of scale are not prohibitive.

The main costs involved in the production of microalgae products arise not in the cultivation, for which there are well-established systems, but in the harvesting and downstream processing of the biomass (Grima et al, 2003). If the potential of microalgae is to be fully realised, several challenges must be overcome, including the development of more efficient downstream processes, which will require a greater understanding of the rheological properties of algal suspensions.

The rheology of suspensions depends on both the nature of the suspended particles (e.g. volume fraction, particle shape) and the interactions between the particles themselves and the fluid flow (Mueller et al, 2009). Suspensions 
of solid particles have been extensively studied and they are well understood and described theoretically, e.g. using Einstein's equation or the KriegerDougherty semi-empirical formula, whereas those of more complex particles, e.g. deformable or active particles such as algae, are less well understood.

Chlorella is one of the most common non-motile genus of microalgae studied rheologically. Wu and Shi (2008) studied Chlorella pyrenoidosa and observed Newtonian behaviour for cell volume fractions of up to $\phi=0.15$, but above this concentration the viscosity increased dramatically and could not be described by Einstein's equation. At higher cell concentrations $(\phi>$ $0.175)$ a yield stress behaviour was observed described by the Herschel-Bulkley model. Zhang et al (2013) examined suspensions of freshwater and marine Chlorella sp. and observed shear-thinning behaviour at all volume fractions ( $\phi=0.08-0.04)$, while Wileman et al (2012) found suspensions of Chorella vulgaris (and suspensions of another non-motile green algae, Nannochloropsis $s p$.) were Newtonian for $\phi<0.02$. They found that another non-motile species, Phaeodactylum tricornutum did not exhibit any non-Newtonian behaviour at any volume fraction examined $(\phi=0.005-0.08)$.

Soulies et al (2013) performed a thorough investigation of suspensions of $C$. vulgaris for a wide range of volume fractions. The cells were roughly spherical with a mean diameter of $1.98 \mu \mathrm{m}$ and were shown to aggregate at high volume and in the absence of flow. Three distinct regimes were identified: a Newtonian one was observed for $\phi \leq 0.115$; a shear thinning one for volume fractions between $\phi=0.115$ and 0.25 , which was attributed to the microstructure of the suspensions; and the formation of flocs at low values of applied stress. A yield stress regime for volumes above 0.25 was observed which was attributed to larger scale aggregate formation. The authors also observed thixotropic-like behaviour in the intermediate and high concentration regimes, which illustrates the rich rheological phenomena of algae suspensions.

The rheology of algal suspensions becomes even more complex when the algae are motile, where the motion of the flagella can have very significant effect on both the microscale and bulk rheology (Foffano et al, 2012; Giomi et al, 2010). The majority of algal blooms in oceans and lakes are motile (around $90 \%$ of strains which produce harmful blooms can swim (Smayda, 1997)), and the motility of green algae has fascinated the fluid dynamics community in recent years (Goldstein, 2015). Flagellated organisms can exhibit two types of swimming behaviour depending on the configuration of their flagella: they can be pullers, i.e. they pull the fluid in front of their body, or pushers, i.e. push the fluid behind their bodies.

Hatwalne et al (2004) analysed the dynamics of active fluids, and predicted that the presence of pushers will lower the bulk viscosity of the suspension, while puller algae will act to increase the viscosity. This has been supported by Sokolov and Aranson (2009), who measured the shear viscosity of suspensions of Bacillus subtilis cells (a pusher species), and found that the viscosity was reduced by a factor of up to 7 , compared to suspensions of non-motile cells. Similarly, Rafaï et al (2010) and Mussler et al (2013) studied the rheology of suspensions of Chlamydomonas reinhardtii, a $10 \mu \mathrm{m}$ puller type microswim- 
mer, and found that the viscosity of suspensions of active cells was higher than that of suspensions of dead cells, as predicted by Hatwalne et al (2004). Using the Krieger-Dougherty rheology model, Rafaï et al (2010) found that the intrinsic viscosity was 4.5 for live cells, but only 2.4 for dead cells. Imaging of cell suspensions revealed different behaviour in a shear flow with the active cells resisting rotation and remaining aligned with the flow nearly $70 \%$ of the time. The authors postulated that the motility may induce shear-thinning behaviour by the motion of the flagella increasing the effective aspect ratio of the cells, or by reducing the cells' ability to rotate in response to the flow at high strain rates. However, a further study by the same group (Mussler et al, 2013) concluded that none of the above two hypotheses can fully describe the observed increase in viscosity.

Adesanya et al (2012) examined the rheology of suspensions of live and dead Scenedesmus obliquus cells, which have a motile phase (Trainor, 1965), and found that when the cells were motile the suspensions had a higher viscosity and exhibited enhanced viscoelastic behaviour. They suggested that this was caused by greater interaction between motile cells, including the tangling of flagella of different cells.

Most of the studies above, with the exception of Wileman et al (2012), have focussed on one type of microalgal species. In most cases a shear thinning behaviour with an increase in concentration has been observed and the effect of motility demonstrated. However, the effect of cell morphology has not been investigated despite the postulation that a hydrodynamic effective aspect ratio effect may be a major factor for flagellated algae. It is not clear, for example, why diatoms such as $P$. tricornutum which exhibit a high aspect ratio are found to maintain Newtonian behaviour Wileman et al (2012). In this study we consider three widely cultivated microalgal strains; we study the rheology of a motile algae species, Tetraselmis chuii, at different volume fractions and compare it to that of Chlorella sp. and Phaeodactylum tricornutum in order to investigate the effects of volume fraction, motility, cell size and morphology.

\section{Materials and methods}

\subsection{Species and culture conditions}

Tetraselmis chuii is an oval (approximately $10 \times 14 \mu \mathrm{m}$ ) chlorophyte (green alga) commonly cultured commercially in the aquaculture industry. Tetraselmis species are highly motile and display four equally sized flagella, found in two pairs (Chengwu and Hongjun, 2002). Chlorella sp. is a spherical chlorophyte which is routinely cultured as a dietary supplement and utilised in bioremediation systems. Phaeodactylum tricornutum is a non-motile, unicellular diatom species which can display an oval or triradiate morphology under culture, but most commonly exists in fusiform morphology approximately $4 \times 10 \mu \mathrm{m}$ in size (Tesson et al, 2009). A model diatom, Phaeodactylum is one of the first microalgae to have its genome sequenced, and has recently been genetically modified 
to optimise omega-3 nutraceutical production (Hamilton et al, 2014). The selection of these strains, therefore, allows comparisons to be made of similar sized and/or shaped, unicellular algae displaying both motile and non-motile characteristics, which are already or are becoming established in a variety of industrial processes.

Tetraselmis chuii (CCAP 8/6), Chlorella sp. (CCAP 211/53) and Phaeodactylum tricornutum (CCAP 1052/1A) were obtained from Culture Collection of Algae and Protozoa, SAMS. Microalgal cultures were grown in F/2 medium (Guillard and Ryther, 1962) at $18^{\circ} \mathrm{C}, 16: 8$ (light:dark) cycle and 30 $\mu \mathrm{mol} / \mathrm{m}^{2} \mathrm{~s}^{2}$ irradiance in 3.5 or $10 \mathrm{~L}$ bubble column photobioreactors (PML, UK). F/2 medium was made using an aquatic salt mix (GroTech coral marine easy mix) to $32 \mathrm{ppt}$ and the addition of F/2 nutrients (Cell-hi F2P, VariconAqua, UK). Microalgae were harvested by centrifugation at $200 \mathrm{~g}$ (3000 $\mathrm{rpm}$ ) for $3 \mathrm{~min}$ in an Octafuge VI centrifuge and re-suspended in phosphate buffered saline (PBS) at the desired volume fraction (either $\phi=0.05,0.1$, 0.15 or 0.2 ). A homogeneous sample of the algae suspension was achieved by vigorous shaking.

Figure 1 shows cell images to illustrate the cell morphology and relative sizes. In order to quantify the cell size distributions, cell images were acquired and dimension information was extracted using ImageJ (Schneider et al, 2012). The results are shown in Table 1 and the distributions of the major cell diameter and aspect ratio in Figure 2.

In order to investigate the effect of cell motility, it was desirable to be able to compare motile and non-motile suspensions of the same strain. $T$. chuii cells become increasingly non-motile in stationary phase, presumably due to lack of energy (light inhibition in dense cultures) and the availability of nutrients. The reduced motility ('non-motile') suspension tested (at $\phi=$ 0.1 ) was sampled from a stationary phase culture and reduced motility was confirmed by observation under the microscope. It was shown that even though the cell arrangement was extremely crowded, some cells were still capable of spinning around, albeit it less fervently.

\subsection{Rheology measurements}

Rheological characterisation of algae suspensions was performed using a rotational ARES rheometer (TA Instruments, New Castle, DE, USA) using a Couette cell geometry. Steady Rate Sweep tests were performed for shear rates ranging from 20 to $200 \mathrm{~s}^{-1}$ at room temperature and volume fractions ranging from $5 \%$ to $20 \%$.

During testing, the inner drum of the Couette cell rotates at a range of pre-set speeds to provide the shear deformation to the algae sample. A shear rate is thus generated and applied to the algae suspension. The torque exerted by the rotating sample is detected by a transducer and converted to a wall shear stress. The viscosity of the algae suspension is then obtained by: 


$$
\eta_{\mathrm{eff}}=\frac{\tau}{\dot{\gamma}},
$$

where $\eta_{\text {eff }}$ is the effective dynamic viscosity [Pa s] of the suspension, $\dot{\gamma}$ the measured shear stress $[\mathrm{Pa}]$ and $\dot{\gamma}$ the applied shear rate $\left[\mathrm{s}^{-1}\right]$.

The inner drum has a diameter of $r_{1}=8.25 \mathrm{~mm}$ and the outer cylinder has a diameter of $r_{2}=8.5 \mathrm{~mm}$, leaving a radial gap of $d=0.25 \mathrm{~mm}$. The depth of the fluid in the drums was $13.5 \mathrm{~mm}$. The Reynolds number is defined as

$$
R e=\frac{\rho \omega r_{1} d}{\eta_{\mathrm{eff}}},
$$

where $\rho$ is the density of the fluid and $\omega$ is the rotational velocity.

When the Reynolds number exceeds a critical value, the flow becomes unstable (Esser and Grossmann, 1996), causing Equation 1 to break down and the viscosity estimates to become unreliable. The maximum rotational velocity employed in the experiments was $5.8 \mathrm{rad} / \mathrm{s}$, and the lowest effective viscosity encountered in any measurement was $0.89 \mathrm{mPa}$ s. Assuming the density of the suspensions was $1000 \mathrm{~kg} / \mathrm{m}^{3}$, the largest Reynolds number encountered in any experiment was $R e_{\max }=53.4$.

The critical Reynolds number at which the flow becomes unstable is given by

$$
R e_{c}=\frac{1}{0.1556^{2}} \frac{(1+\beta)^{2}}{2 \beta \sqrt{(1-\beta)(3+\beta)}},
$$

where $\beta=r_{1} / r_{2}=0.9706$ (Esser and Grossmann, 1996). This corresponds to a critical $R e_{c}$ of 241.8 , which is almost an order of magnitude larger than that encountered in the current study (even allowing for increases in the density of the solutions due to the addition of algae cells), indicating that our results are not affected by flow instabilities and remain valid at high strain rates.

As with all suspensions, the rheology measurements are susceptible to wall slip, whereby the particles or cells tend to migrate away from the walls of the rheometer, resulting in a low viscosity layer forming near the wall and reducing the apparent viscosity of the suspension. The wall slip phenomena have been reviewed by a number of authors, e.g. Barnes (1995) and Hatzikiriakos (2015). Despite having a potentially very significant effect, this factor is typically ignored (Buscall, 2010) or eliminated in rheological studies by modifying wall roughness. Nevertheless, as Soulies et al (2013) note, wall slip is equally likely to occur in industrial contexts such as photobioreactors and downstream processing systems, and is an inherent mechanism by which algal suspensions respond to shear. To evaluate the presence and extent of wall slip typically requires the ability to image the fluid as it is being sheared in the rheometer (Soulies et al, 2013) which is often not feasible, or the use of microfluidic approaches to image suspension flows under shear; the latter approach has been followed in blood flow studies (Sherwood et al, 2012) as the cell depleted layer is a key feature in the microcirculation. It is clear that more work is required to understand the mechanism of slip and this is beyond the scope of this study. 


\section{Results}

\subsection{Rheology of Tetraselmis chuii suspensions}

Figure 3 shows the measured shear stress and effective viscosity profiles of suspensions of $T$. chuii for various volume fractions. It is clear that the presence of $T$. chuii cells causes an increase in the observed stress (Figure 3(a)) and the effective viscosity (Figure $3(\mathrm{~b}))$ of the fluid. The pure PBS $(\phi=0)$ shows Newtonian behaviour with shear stress increasing linearly with strain rate and the effective viscosity remaining constant. However, when T. chuii cells are suspended within the fluid, the effective viscosity falls with increasing strain rate, indicating shear-thinning behaviour. This non-Newtonian behaviour becomes progressively more pronounced as the cell concentration is increased; at $\phi=0.05$, the suspension displays predominantly Newtonian-like properties, whereas from $\phi=0.1$ onwards, non-Newtonian behaviour emerges and shear-thinning can be clearly observed, particularly at $\dot{\gamma}<50 \mathrm{~s}^{-1}$.

In order to characterise this shear thinning effect and identify how it varies with volume fraction, the experimental data in Figure 3(a) was fitted to the Herschel-Bulkley model:

$$
\tau=\tau_{y}+K \dot{\gamma}^{n}
$$

where $\tau_{y}$ is the yield stress $[\mathrm{Pa} \mathrm{s}], \mathrm{K}$ the consistency $\left[\mathrm{Pa} \mathrm{s}{ }^{n}\right.$ ] and $n$ the flow index, with $n<1$ for shear-thinning fluids. When the yield stress is exceeded, the viscosity of a Herschel-Bulkley fluid is given by:

$$
\eta=K|\dot{\gamma}|^{n-1}+\tau_{y}|\dot{\gamma}|^{-1}
$$

It should be noted that even in the case of fluids that exhibit quasiNewtonian behaviour ( $n \approx 1)$, the effective viscosity will be greater at low strain rates if the yield stress is greater than zero (due to the $\tau_{y}|\dot{\gamma}|^{-1}$ term).

The estimated Herschel-Bulkley parameters found are listed in Table 2, and the solid lines in Figures 3(a) and 3(b) represent the corresponding estimates of the shear stress and effective viscosity (Equations 4 and 5), respectively. The uncertainties in the estimates of the Herschel-Bulkley parameters are represented by their standard deviations, which are also listed in Table 2. There is some variation in the estimated values of the yield stress, which may be a result of the limited number of measurements at low strain rates. The flow index is approximately equal to unity for $\phi<0.1$. As the T. chuii concentration is increased further, $n$ declines, indicating progressive shearthinning behaviour, while the yield stress is also reduced and the consistency increases. These results are broadly consistent with the studies of Wu and Shi (2008), Wileman et al (2012) and Soulies et al (2013), who observed Newtonian behaviour in suspensions of various Chlorella species at low volume fractions, and shear-thinning behaviour above a critical value in the range $\phi=0.02-$ 0.15 . 
The shear-thinning nature of algae suspensions has been reported in the 1 literature (Rafaï et al, 2010; Adesanya et al, 2012; Soulies et al, 2013) and is a well known aspect of particle suspensions. It is attributed to cell interactions and microstructural changes of the suspension (Adesanya et al, 2012; Soulies et al, 2013) or to highly localised viscous heating of the suspension at high volume fractions (Mueller et al, 2009). Soulies et al (2013) reported the existence of flocs in the shear thinning regime for Chlorella suspensions both with and without shearing. In order to investigate the presence of flocculation, suspensions of $T$. chuii at $\phi=0.05$ were tested in the rheometer at different shear rates applied for 20 seconds each and samples were studied under the microscope post-shearing (Figure 4). Some cell aggregation was observed in the micrographs obtained at $10 \mathrm{~s}^{-1}$, which might explain the shear thinning behaviour at low shear rates (Figure 4(b)), whereas no cell aggregation was evident at high shear rates (Figures $4(\mathrm{c})$ and $4(\mathrm{~d})$ ). It should be noted that these images were taken after the shearing had stopped and it was not possible to acquire images in situ.

The rheological properties of suspensions of solid spherical particles in the dilute regime (i.e. $\phi \leq 0.05$ ) have been characterised by Einstein's equation:

$$
\eta=\eta_{0}(1+\alpha \phi)
$$

where $\eta_{0}$ is the viscosity of the suspending medium, and $\alpha$ is the intrinsic viscosity ( $\alpha=2.5$ for passive, rigid, spherical particles). The model is known to work well in the dilute regime, whereas at higher concentrations the KriegerDougherty model is often used (Mueller et al, 2009):

$$
\eta=\eta_{0}\left(1-\frac{\phi}{\phi_{m}}\right)^{-\alpha \phi_{m}}
$$

where $\phi_{m}$ is the maximum packing volume fraction. This factor is often treated as a free variable that can be fitted to experimental data. However, given the limited number of data points at low concentrations, we take $\phi_{m}=0.62$, following the approach of Rafai et al (2010).

Figures 5(a) and 5(b) compare the experimental data to Einstein's equation and the Krieger-Dougherty model, respectively, for a range of strain rates. As can be seen, both equations fail to match the observed viscosity at all strains for $\phi>0.1$ (in each case $\alpha$ was found by applying the best-fit to the data for $\phi \leq 0.1$ ). The breakdown of the Krieger-Dougherty model is particularly evident at the highest volume fraction. On the basis of solid particle suspension theory (Mueller et al, 2009), the volume fraction presented here $(\phi=0.05-0.2)$ corresponds to the semi-dilute regime, and the poor performance of Equations 6 and 7 is well known.

It is interesting to note that the best-fit lines of the Einstein and KriegerDougherty equations in Figure 5 are not the same at all strain rates, i.e. the intrinsic viscosity varies with $\dot{\gamma}$. In order to examine this dependence, the parameters were evaluated for both equations at a range of strain rates, and are presented in Figure 6 . The trends are qualitatively the same: $\alpha$ declines linearly 
for $\dot{\gamma}<100 \mathrm{~s}^{-1}$, at higher strain rates it also declines linearly but at a less steep gradient. This indicates that the degree to which the cell concentration affects the rheology is dependent on the strain rate: at low strain rates the rheology is strongly dependent on the cell concentration (i.e. $\alpha$ is high), while at high strain rates the concentration has a weak effect on the viscosity (low $\alpha$ ). The clear change in the dependency of $\alpha$ on $\dot{\gamma}$ at $\dot{\gamma}=100 \mathrm{~s}^{-1}$ suggests that there is a change in the physical mechanism by which the suspended cells affect the fluid rheology. It seems likely that this is related to the appearance of flocs at low shear rates (Figure 4(b)), and their absence in the micrographs acquired for $\dot{\gamma} \geq 100 \mathrm{~s}^{-1}$ (Figures 4(c) and 4(d)), as the tendency to form flocs at low strain rates induces a significant increase in the observed viscosity. Other potential factors include the presence of extracellular polysaccharides and cell deformability.

The Krieger-Dougherty model and the Einstein equation were developed for suspensions of passive, rigid spheres. However, the T. chuii cells are neither passive or rigid; they are motile and can respond to the flow, thereby affecting the rheology. In order to study this effect, the rheological characteristics of suspensions of motile and non-motile $T$. chuii cells were measured.

Figure 7(a) compares the variation in the measured stress with strain rate, for motile and non-motile suspensions at different volume fractions, with the corresponding viscosity profiles shown in Figure 7(b). It is clear that the motility of cells is associated with a significant increase in viscosity.

The data in Figure 7(a) was fitted to the Herschel-Bulkley model, and the lines in Figures 7(a) and 7(b) correspond to the estimated stress and viscosity profiles (Equations 4 and 5, respectively). The estimates of the consistency and flow index for each case are presented in Figure 8(a) and 8(b), respectively. As noted previously, increasing the concentration of motile $T$. chuii cells causes a dramatic increase in the consistency of the suspension and a drop in the flow index (i.e. the suspension exhibits increasing shear-thinning behaviour). In contrast, when the $T$. chuii cells are non-motile, both $K$ and $n$ remain largely constant as the volume fraction is increased. The flow index remains at $n \approx 1$ (with some scatter due to the fitting process), indicating that the addition of non-motile cells does not lead to significant shear-thinning behaviour (although the effective viscosity may be larger near $\dot{\gamma}=0$ due to the effects of the yield stress).

The fact that the cell motility increases the bulk viscosity of the suspension implies that the $T$. chuii cells align to resist the flow, or the motion of the flagella may affect rheology by changing the effective aspect ratio of the cells. The latter effect will be discussed in Section 4. This behaviour is typical of puller type swimming algae (Hatwalne et al, 2004; Rafaï et al, 2010). This information suggests that when processing $T$. chuii suspensions in industrial contexts, it may be beneficial to induce a reduction in motility by UV radiation exposure or chemical treatment, thereby lowering the viscosity, and reducing the energy requirements.

Meanwhile, the different trends in Figure 8(b) suggest that the pronounced shear-thinning behaviour is a direct result of the cell motility; at high strain 
rates the cells cannot align to resist the flow (and increase the viscosity) as effectively as at lower strain rates.

\subsection{Effect of algal species}

In order to understand the role of $T$. chuii morphology on the suspension rheology, we also examined the rheology of suspensions of the different algal species described in Section 2.1. A Phaeodactylum tricornutum suspension was examined at a volume fraction of $\phi=0.1$, and suspensions of Chlorella sp. were examined at $\phi=0.1$ and 0.2 . The variations in the stress and effective viscosity for each case are presented in Figure 9.

The Phaeodactylum suspension shows clear shear-thinning behaviour (Figure 9(a)), with a viscosity profile quite similar to that of the T. chuii suspension at $\phi=0.1$. Wileman et al (2012) did not observe any non-Newtonian behaviour at slightly lower volume fractions $(\phi=0.005-0.08)$, which suggests that there is a critical value at which suspensions of $P$. tricornutum become non-Newtonian, as has been observed for Chlorella (Wileman et al, 2012; Soulies et al, 2013; Wu and Shi, 2008).

The Chlorella suspensions exhibit some particularly interesting behaviour. Figures 9(a) and 9(b) show that, similar to other cases, the viscosity falls dramatically with strain rate, as occurs for shear-thinning fluids. This is in agreement with the work of Zhang et al (2013), examining suspensions of Chlorella sp., and the work of Soulies et al (2013), examining suspensions of Chlorella vulgaris, who found that the rheology was shear-thinning at $\phi=$ 0.165 , but Newtonian (or very weakly shear-thickening) at $\phi=0.082$. However, upon closer inspection of the variation in shear stress (Figures 9(a) and 9(b)), it is apparent that the suspension is in fact shear-thickening, as the rate of increase in stress with strain is greater at high strain rates. This can be seen in Figure 9(c) for $\dot{\gamma}>100 \mathrm{~s}^{-1}$. It has been noted previously that the viscosity of many suspensions initially decreases with strain rate before going through a transition in which it increases (Barnes, 1989; Stickel and Powell, 2005), although this tends to only occur at high cell concentrations.

The parameters of the Herschel-Bulkley model for the Chlorella data are summarised in Table 3. At both volume fractions, the Chlorella suspensions have a relatively large yield stress; this leads to the high effective viscosity observed at low strain rates, via the $\tau_{y}|\dot{\gamma}|^{-1}$ term in the Herschel-Bulkley expression for $\eta$ (Equation 5). The point at which the effective viscosity begins to increase with strain rate is found by differentiating Equation 5, and is equal to

$$
\dot{\gamma}_{\text {crit }}=\left(\frac{\tau_{y}}{K(n-1)}\right)^{1 / n} .
$$

These values are listed in Table 3 , and are consistent with the measurements presented in Figures 9(b) and 9(d) (and shown in detail in Figure 10(a)). At low strain rates, the yield stress term in Equation 5 is very large, and the 
shear thinning effects are not clearly visible. However, it is clear from Figure 10(b) that the shear stress has a super-linear $(n>1)$ dependence of strain rate. This super-linearity is not a result of uncertainties in the fitting process, as for both Chlorella suspensions, the standard deviation in the estimates of the flow index is small (Table 3 ).

\section{Discussion}

It was shown in Figure 6 that the estimates of the intrinsic viscosity of $T$. chuii suspensions vary considerably with strain rate; over the range examined $\left(\dot{\gamma}=20-200 \mathrm{~s}^{-1}\right) \alpha$ decreased by $38 \%$ when calculated using the KriegerDougherty model, and by over $50 \%$ when using the Einstein equation. To the best of the authors' knowledge, the strain-dependence of the intrinsic viscosity of algae suspensions has not previously been noted in the literature. However, it may explain some of the variation in the intrinsic viscosity measured in previous studies. Zhang et al (2013) reported intrinsic viscosities of 24.7 and 16.1 for dilute $(\phi \leq 0.04)$ suspensions of freshwater and marine Chlorella sp., respectively, found using the Krieger-Dougherty model at a strain rate of 6 $\mathrm{s}^{-1}$. Soulies et al (2013) studied Chlorella vulgaris, and chose to fit their data to the Quemada (1998) model, which is the same as Equation 7, with the exponent set to -2 , i.e. $\alpha \phi_{m}=2$. Using this approach and measurements at high strains rates $\left(\dot{\gamma} \approx 50-500 \mathrm{~s}^{-1}\right)$, they found $\phi_{m}=0.637$, which corresponds to $\alpha=3.14$. The difference of almost an order of magnitude between the estimates of the intrinsic viscosity found in the two studies may arise from the different algae species or the differing experimental conditions, such as the greater presence of polymeric material in the experiments of Zhang et al (2013) (who used Bold's basal and F/2 medium for the freshwater and marine suspensions, respectively, while Soulies et al (2013) used Hunter's solution). However, the observation of higher intrinsic viscosity estimates at low strain rates in these studies is consistent with the results shown in Figure 6, where the variation in $\alpha$ cannot be attributed to changes in the suspending medium, and indicates that the intrinsic viscosity of algal suspensions may be inherently strain rate-dependent.

An interesting finding of this study is evidence of shear-thickening behaviour in Chlorella sp. suspensions at high strain rates (Figure 10), which could not be attributed to uncertainties in the estimation of the flow index (Table 3), and has not previously been reported in the literature. Zhang et al (2013) examined the rheology of suspensions of Chlorella sp., but presented only the variation in effective viscosity rather than the shear stress, making it difficult to ascertain whether the high viscosity they observed at low strain rates was a result of shear-thinning behaviour, or a result of a high yield stress. However, our findings are clearly in contrast to those of Soulies et al (2013) and Wileman et al (2012), who found Chlorella suspensions were strongly shear-thinning for $\phi>0.1$. 
It is not clear why the opposite trend is observed in this work. A possible factor may relate to the presence of extracellular polysaccharides (EPS) into the suspending medium. As a chlorophyte, Chlorella is a strong producer of starch (Bailey and Neish, 1954), and starch solutions are well known to have shear-thickening rheology (Barnes et al, 1989); thus if the starch were to somehow enter the suspending medium, perhaps as a result of cell damage at high strain rates, this could explain the clear shear-thickening behaviour observed here. However, Kaplan et al (1987) found that after several days, only about $10 \%$ of the polysaccharide produced by Chlorella stigmatophora dissolved into the suspending medium, with the rest remained bound to the cell. Alternatively, the shear-thickening behaviour here may be a result of thixotropic behaviour, as was observed in the study of Soulies et al (2013). Further work is planned to investigate these possibilities.

The measurements of the suspensions of three different algae species allows the effect of cell morphology to be examined. Non-spherical particle suspensions behave differently to spherical ones; hydrodynamic and inter-particle interactions are different and more importantly non-spherical particles are capable of orienting themselves with the flow (Mueller et al, 2009) rather than rotating freely as spheres do. Genovese (2012) showed that for a given volume fraction the relative viscosity of non-spherical particles increases with increasing aspect ratio due to extra energy dissipation. Of the non-motile algal suspensions presented in Figure 9, the most viscous suspension is that of P. tricornutum, which has the largest aspect ratio $\left(r_{p}=6.5\right.$, Table 1$)$, and the least viscous is that of Chlorella, which has the lowest aspect ratio $\left(r_{p}=1\right)$, suggesting a link between $r_{p}$ and viscosity. Rafaï et al (2010) argued that the difference in the viscosity of motile and dead $C$. rheihardtii suspensions was caused by the moving flagella increasing the effective aspect ratio of the cells. The data of the motile and non-motile $T$. chuii cells, in conjunction with the other non-motile species, allows us to assess the role of motility and aspect ratio on the suspension rheology.

Figure 11 shows the intrinsic viscosity of the different suspensions as a function of the cell aspect ratio, at $\dot{\gamma}=60 \mathrm{~s}^{-1}$ and $\phi=0.1$. Examining the non-motile cases (black symbols), there appears to be an approximate relationship of increasing intrinsic viscosity with aspect ratio, as was found by Mueller et al (2009) (for solid spheres, using the Quemada (1998) model) . The motile $T$. chuii suspension has a significantly higher intrinsic viscosity than the non-motile cases at a similar aspect ratio. If the only way by which the flagella (and the ability of the $T$. chuii cells to move) affects the suspension rheology is through an increase in the effective aspect ratio of the cells, then the trend in Figure 9 indicates that the T. chuii cells would be required to have an effective aspect ratio in the approximate range $6-8$. The $T$. chuii flagella are approximately $12 \mu \mathrm{m}$ in length, and are all positioned at the same point on the cell. If they are aligned to maximise the aspect ratio, the effective value is still only $r_{p, \max }=(14.63+12) / 9.591=2.78$ (Table 1). This is shown in Figure 11 (open circle) and is still significantly above the trend line predicted by the non-motile cases. This indicates that while it is likely that the aspect ratio 
plays a role in determining the suspension rheology, the increase in viscosity associated with the cell motility cannot be attributed to an increase in the effective aspect ratio alone. Other mechanisms by which the motility may increase the viscosity include the greater diffusion of EPS into the suspending medium in suspensions of live compared to dead cells, flocculation and the preferential alignment of cells to oppose the flow, as discussed earlier.

\section{Conclusions}

The rheology of suspensions of three algal strains, Tetraselmis chuii, Chlorella sp. and Phaeodactylum tricornutum, was examined using a rotational rheometer for a range of volume fractions and strain rates. The measurements were fitted to the Herschel-Bulkley model, while the effect of volume fraction on the T. chuii suspensions was modelled using the Krieger-Dougherty and Einstein equations, which both performed well for $\phi \lesssim 0.1$. The intrinsic viscosity was found to decrease with strain rate, indicating that the rheology became progressively less sensitive to the concentration of algal cells as the strain rate was increased. The intrinsic viscosity of the cells declined linearly for $\dot{\gamma} \leq 100$ $\mathrm{s}^{-1}$, where there was an inflection point, and at high strain rates it continued to decline linearly but with a slower gradient. Micrographs of the $\phi=0.05$ suspension acquired shortly after the viscosity measurements showed signs of flocculation in the $\dot{\gamma}=10 \mathrm{~s}^{-1}$ case, but none in the $\dot{\gamma}=100 \mathrm{~s}^{-1}$ case, suggesting that the change of behaviour in the intrinsic viscosity at $100 \mathrm{~s}^{-1}$ may be related to presence/absence of flocculation. Other possible causes include the presence of extracellular polysaccharides and the deformability of the T. chuii cells.

The Herschel-Bulkley model clearly indicated that the T. chuii suspensions were shear-thinning $(n<1)$. However, suspensions of non-motile T. chuii cells did not show any signs of shear-thinning (i.e. $n$ was approximately equal to unity at all volume fractions), indicating that it is the motility of the cells that is the cause of this non-Newtonian behaviour. In contrast, the Chlorella suspensions indicated shear-thickening behaviour $(n>1)$. However, the yield stress of the suspensions meant that the effective viscosity remained high at low strain rates, and only started to increase with strain rate at $\dot{\gamma} \gtrsim 156 \mathrm{~s}^{-1}$.

Finally, the viscosity profiles of all three algal strain suspensions were compared. It was found that the viscosity tended to increase with the aspect ratio of the algal cells, i.e. Phaeodactylum suspensions $\left(r_{p}=6.47\right)$ were the most viscous, while Chlorella suspensions $\left(r_{p}=1\right)$ were the least so. It has been speculated in the literature that the increased viscosity of motile suspensions may be caused by an increase in the effective aspect ratio of the cells, due to the motion of the flagella (Rafaï et al, 2010). In order to assess to what extent this is the case for the $T$. chuii suspensions, the viscosity of each strain were compared as a function of $r_{p}$. It was shown that even if the flagella were fully extended, the increase in aspect ratio is not sufficient to account for the 
observed increase in viscosity. Therefore, the increased viscosity of motile $T$. chuii cells requires that the cells preferentially align to resist the flow.

Acknowledgements This work was partially supported by a grant awarded from the Innovate UK (formally known as Technology Strategy Board) (TSB 4783-44269). The authors would like to thank Dr Efstathios Kaliviotis for helpful discussions regarding the rheometer measurements.

\section{References}

Adesanya VO, Vadillo DC, Mackley MR (2012) The rheological characterization of algae suspensions for the production of biofuels. J Rheol 56(4):925939

Bailey JM, Neish AC (1954) Starch synthesis in Chlorella vulgaris. Can J Biochem Phys 32(4):452-464

Barnes HA (1989) Shear-thickening ("dilatancy") in suspensions of nonaggregating solid particles dispersed in Newtonian liquids. J Rheol (1978-present) 33(2):329-366

Barnes HA (1995) A review of the slip (wall depletion) of polymer solutions, emulsions and particle suspensions in viscometers: its cause, character, and cure. J Non-Newon Fluid 56:221-251

Barnes HA, F HD, Walters K (1989) An Introduction to Rheology, vol 3. Elsevier

Borowitzka MA (2013) High-value products from microalgae - their development and commercialisation. J Appl Phycol 25:743-756

Borowitzka MA, Moheimani NR (2013) Sustainable biofuels from algae. Mitig Adapt Strategies Glob Chang 18:13-25

Buscall R (2010) Letter to the Editor: Wall slip in dispersion rheology. J Rheol (1978-present) 54(6):1177-1183

Chengwu Z, Hongjun H (2002) Taxonomy and ultrastructure of five species of Tetraselmis (Prasinophyceae) isolated from China seas. Acta Oceanol Sin 21(4):557-579

Esser AE, Grossmann S (1996) Analytic expression for Taylor-Couette stability boundary. Phys Fluids 8(7):1814-1819

Fields CB, Behrenfeld MJ, Randerson P J T Falkowski (1998) Primary production of the biosphere: integrating terrestrial and oceanic components. Science 281(5374):237-240

Foffano G, Lintuvuori JS, Morozov AN, Stratford K, Cates ME, Marenduzzo D (2012) Bulk rheology and microrheology of active fluids. Euro Phys J E $35(98): 1-11$

Genovese DB (2012) Shear rheology of hard sphere, dispersed and aggregated suspensions, and filler-matrix composites. Adv Colloid and Interfac 171:1-16

Giomi L, Liverpool TB, Marchetti MC (2010) Sheared active fluids: Thickening, thinning, and vanishing viscosity. Phys Rev E 81(5):051,908

Goldstein RE (2015) Green algae as model organisms for biological fluid dynamics. Annu Rev Fluid Mech 47:343 
Grima EM, Belarbi EH, Fernández FA, Medina AR, Chisti Y (2003) Recovery of microalgal biomass and metabolites: process options and economics. Biotechnol Adv 20(7):491-515

Hatwalne Y, Ramaswamy S, Rao M, Simha RA (2004) Rheology of activeparticle suspensions. Phys Rev Lett 92(11):118,101

Hatzikiriakos SG (2015) Slip mechanisms in complex fluid flows. Soft Matter 11(40):7851-7856

Kaplan D, Christiaen D, Arad S (1987) Chelating properties of extracellular polysaccharides from Chlorella spp. Appl Environ Microb 53(12):2953-2956

Mueller S, Llewellin EW, Mader HM (2009) The rheology of suspensions of solid particles. In: P R Soc A, The Royal Society, p rspa20090445

Mussler M, Rafaï S, Peyla P, Wagner C (2013) Effective viscosity of nongravitactic Chlamydomonas reinhardtii microswimmer suspensions. EPLEurophys Lett 101(5):54,004

Quemada D (1998) Rheological modelling of complex fluids. I. The concept of effective volume fraction revisited. Eur Phys J-Appl Phys 1(1):119-127

Radakovits R, Jinkerson RE, Darzins A, Posewitz MC (2010) Genetic engineering of algae for enhanced biofuel production. Eukaryot Cell 9(4):486-501

Rafaï S, Jibuti L, Peyla P (2010) Effective viscosity of microswimmer suspensions. Phys Rev Lett 104(9):098,102

Schneider CA, Rasband WS, Eliceiri KW (2012) NIH Image to ImageJ: 25 years of image analysis". Nat Methods 9:671-675

Sherwood JM, Dusting J, Kaliviotis E, Balabani S (2012) The effect of red blood cell aggregation on velocity and cell-depleted layer characteristics of blood in a bifurcating microchannel. Biomicrofluidics 6(024119)

Smayda TJ (1997) Harmful algal blooms: Their ecophysiology and general relevance to phytoplankton blooms in the sea. Limnol Oceanogr 42(5.2):11371153

Sokolov A, Aranson IS (2009) Reduction of viscosity in suspension of swimming bacteria. Phys Rev Lett 103(14):148,101

Soulies A, Pruvost J, Legrand J, Castelain C, Burghelea TI (2013) Rheological properties of suspensions of the green microalga Chlorella vulgaris at various volume fractions. Rheol Acta 52(6):589-605

Stickel JJ, Powell RL (2005) Fluid mechanics and rheology of dense suspensions. Annu Rev Fluid Mech 37:129-149

Trainor FR (1965) Motility in Scenedesmus cultures incubated in nature. B Torrey Bot Club 92(5):329-332

Walker DA (2009) Biofuels, facts, fantasy, and feasibility. J Appl Phycol 21(5):509-517

Wileman A, Ozhan A, Berberoglu H (2012) Rheological properties of algae slurries for minimizing harvesting energy requirements in biofuel production. Bioresource Technol 104:432-439

Wu Zy, Shi Xm (2008) Rheoogical properties of Chlorella pyrenoidsa culture grown heterotrophically in a fermentor. J Appl Phycol 20(3):279-282

Zhang X, Jiang Z, Chen L, Chou A, Yan H, Zou YY, Zhang X (2013) Influence of cell properties on rheopogical characterization of microalgae suspensions,. 
Bioresource Technol 139:209-213 

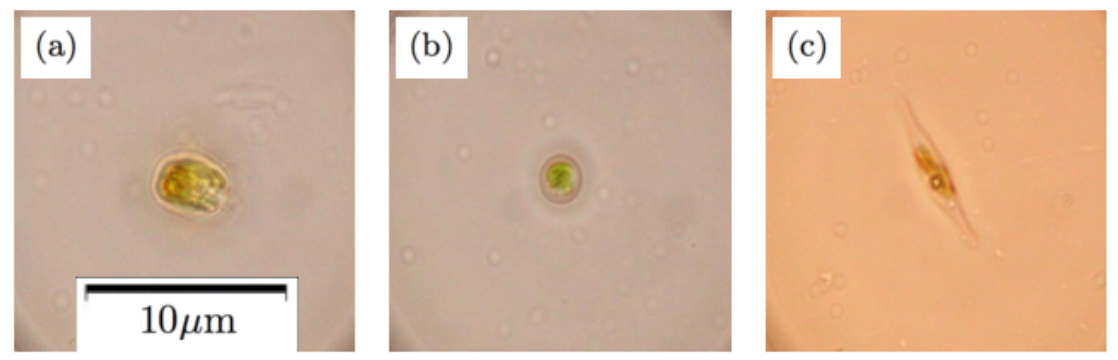

Fig. 1 Images of isolated cells illustrating cell morphology: T.chuii (a); C. sp. (b); and P. tricornutum (c).

Table 1 Cell sizes of samples tested.

\begin{tabular}{llll}
\hline Dimension & T. chuii & C. sp. & P. tricornutum \\
\hline Average Major Diameter $[\mu \mathrm{m}]$ & $14.63 \pm 0.574$ & $6.523 \pm 2.422$ & $23.968 \pm 4.995$ \\
Average Minor Diameter $[\mu \mathrm{m}]$ & $9.591 \pm 1.955$ & & $3.817 \pm 1.123$ \\
Aspect Ratio, $r_{p}$ & $1.539 \pm 0.312$ & & $6.473 \pm 2.514$ \\
\hline
\end{tabular}

Table 2 Yield stress, consistency and flow index found for different concentrations of motile T. chuii, and the standard deviation of the estimates.

\begin{tabular}{llllll}
\hline$\phi$ & 0 & 0.05 & 0.1 & 0.15 & 0.2 \\
\hline$\tau_{y}[\mathrm{mPa}]$ & 5.48 & 32.39 & 19.74 & 12.31 & 0 \\
$\sigma\left(\tau_{y}\right)[\mathrm{mPa}]$ & 0.006 & 0.009 & 0.01 & 0.008 & $1 \times 10^{-4}$ \\
$K\left[\mathrm{mPa} \mathrm{s}^{n}\right]$ & 0.804 & 1.15 & 5.1 & 7.82 & 12.95 \\
$\sigma(K)\left[\mathrm{mPa} \mathrm{s}^{n}\right]$ & $2 \times 10^{-4}$ & $4.9 \times 10^{-4}$ & 0.001 & 0.001 & 0.001 \\
$n$ & 1.01 & 0.998 & 0.781 & 0.762 & 0.69 \\
$\sigma(n)$ & 0.043 & 0.075 & 0.047 & 0.026 & 0.02 \\
\hline
\end{tabular}

Table 3 Hershcel-Bulkley parameters estimated from suspensions of Chlorella sp. presented in Figures 9(a) and 9(c). The uncertainties were calculated during the fitting process, and correspond to one standard deviation.

\begin{tabular}{lll}
\hline & $\phi=0.1$ & $\phi=0.2$ \\
\hline$\tau_{y}[\mathrm{mPa}]$ & 50.56 & 80.1 \\
$\sigma\left(\tau_{y}\right)[\mathrm{mPa}]$ & 0.005 & 0.002 \\
$K\left[\mathrm{mPa} \mathrm{s}^{n}\right]$ & 0.103 & 0.011 \\
$\sigma(K)\left[\mathrm{mPa} \mathrm{s}^{n}\right]$ & $5 \times 10^{-5}$ & $6 \times 10^{-6}$ \\
$n$ & 1.4 & 1.79 \\
$\sigma(n)$ & 0.085 & 0.08 \\
$\left(\frac{\tau_{y}}{K(n-1)}\right)^{1 / n}\left[\mathrm{~s}^{-1}\right]$ & 156.4 & 158.8 \\
\hline
\end{tabular}


(a) T. chuii

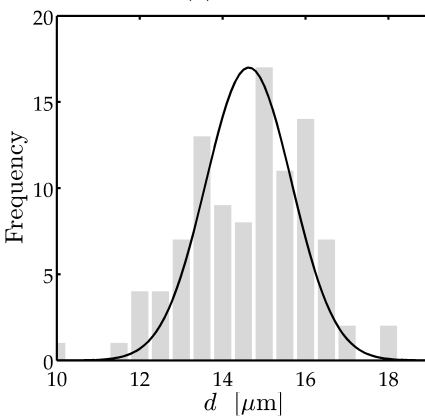

(d) T. chuii

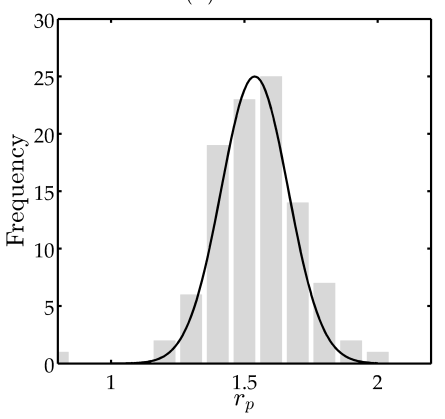

(b) P. tricornutum

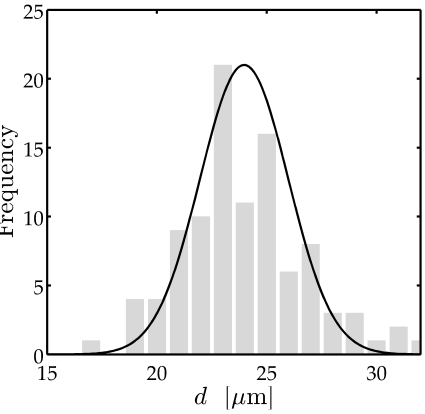

(e) P. tricornutum

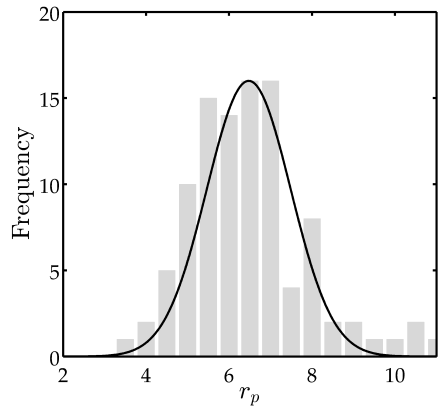

(c) C. sp.

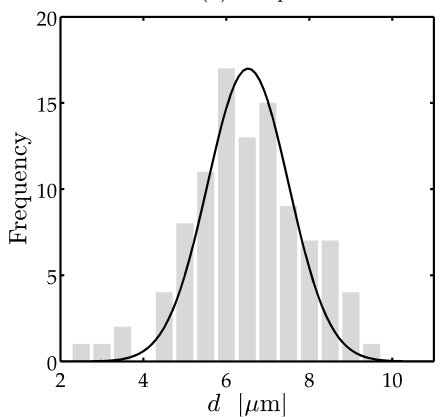

$$
\text { ( }
$$

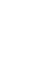


(a)

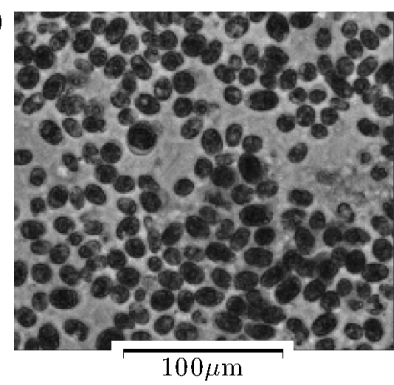

(b)

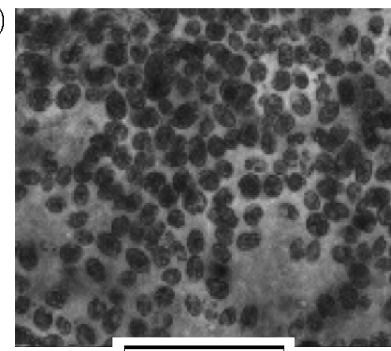

$100 \mu \mathrm{m}$

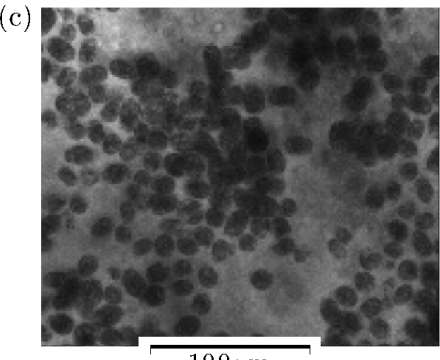

$100 \mu \mathrm{m}$

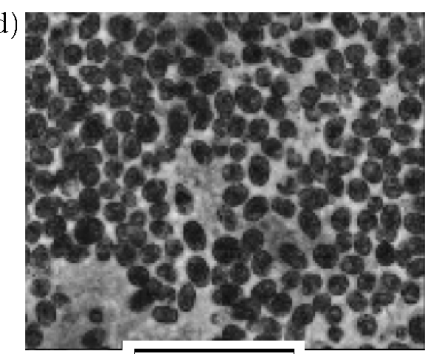

$100 \mu \mathrm{m}$

Fig. 4 Micrograph of $\phi=0.05$ volume fraction Tetraselmis chuii suspension taken with $20 \times$ magnification, $20 \mathrm{~s}$ after the suspension was sheared at rate of $0 \mathrm{~s}^{-1}$ (a), $10 \mathrm{~s}^{-1}$ (b), $100 \mathrm{~s}^{-1}(\mathrm{c})$, and (d) $1000 \mathrm{~s}^{-1}$.
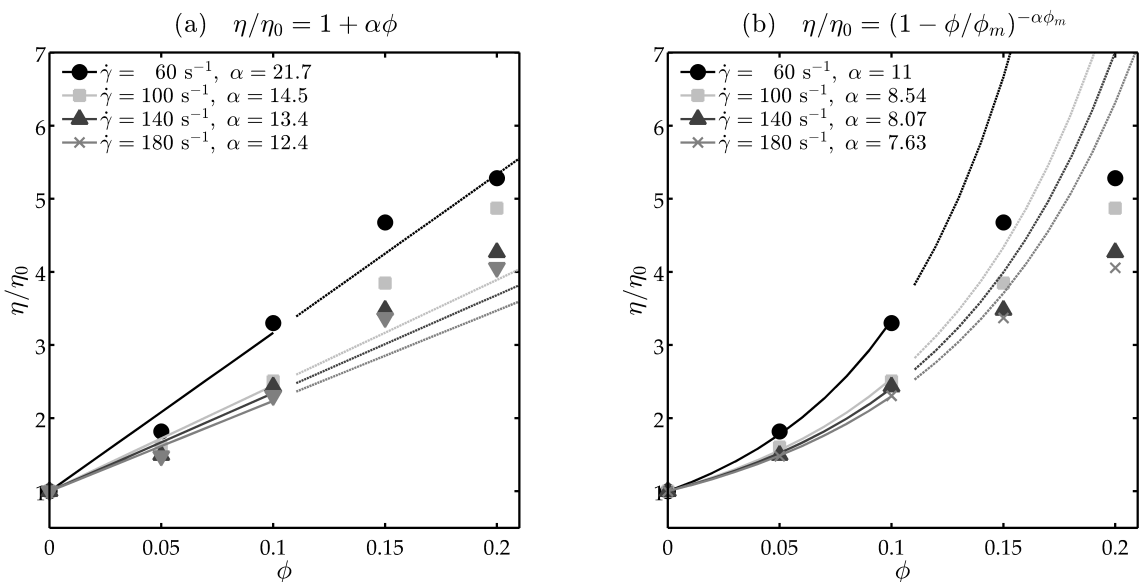

Fig. 5 Variation in effective viscosity of $T$. chuii suspensions as a function of concentration volume, measured at a range of shear rates. The lines in (a) show the best-fit of data to the Einstein's equation (Equation 6), while the lines in (b) represent the best fit to the KriegerDougherty model (Equation 7 ). The estimates of the intrinsic viscosity were calculated using the data for $\phi \leq 0.1$. 


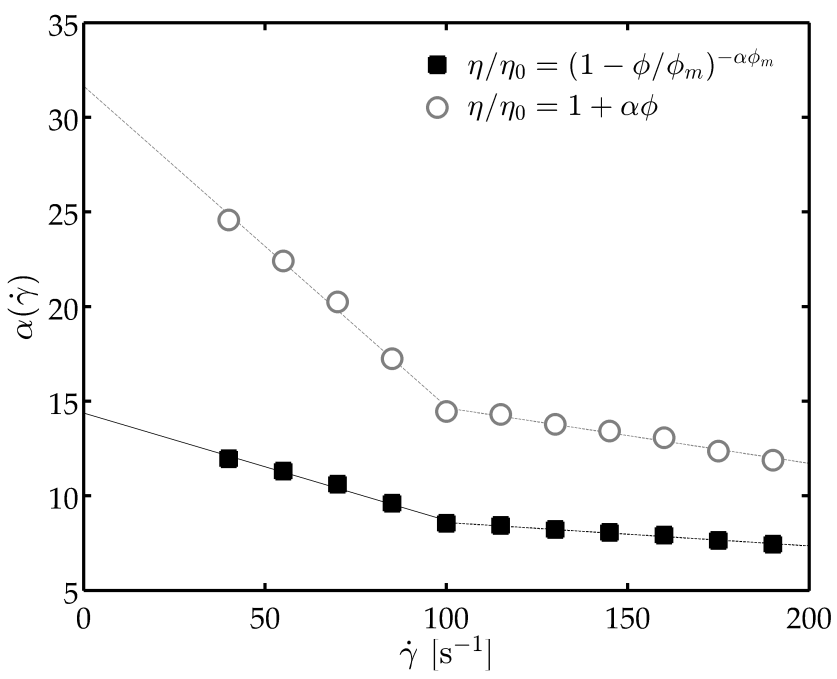

Fig. 6 Variation in the intrinsic viscosity estimated using the Einstein and KriegerDougherty equations with strain rate, for $T$. chuii suspensions.
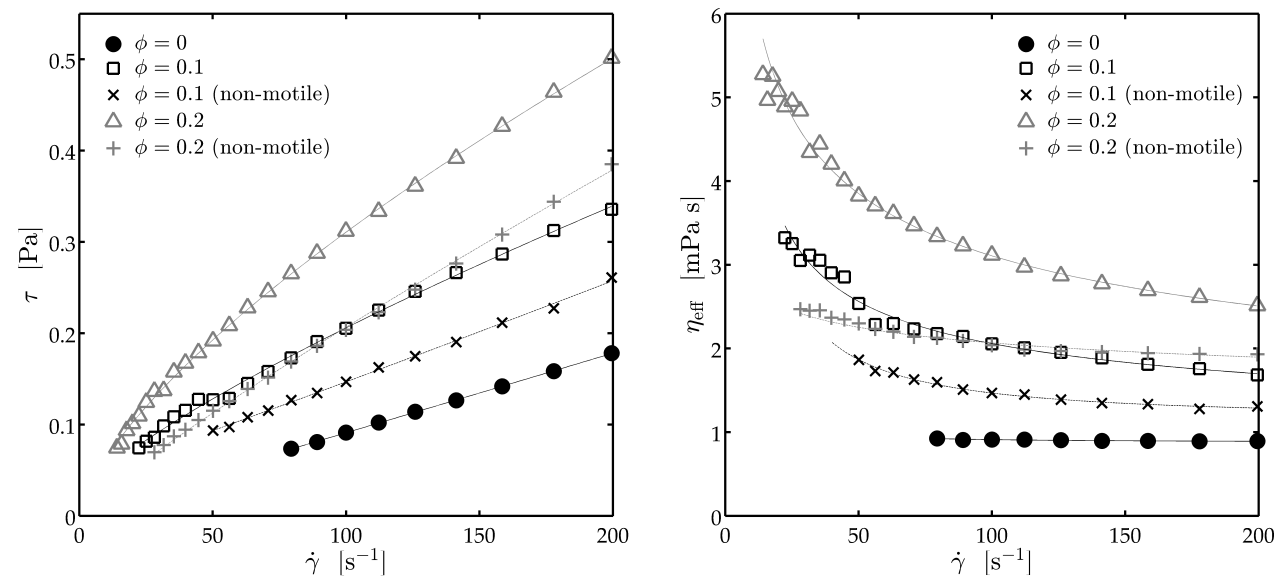

Fig. 7 Shear stress (a) and effective viscosities (b) of PBS and motile and non-motile $T$. chuii suspensions as a function of shear rate. The lines represent the best-fit of the data to the Herschel-Bulkley model (Equations 4 and 5). 

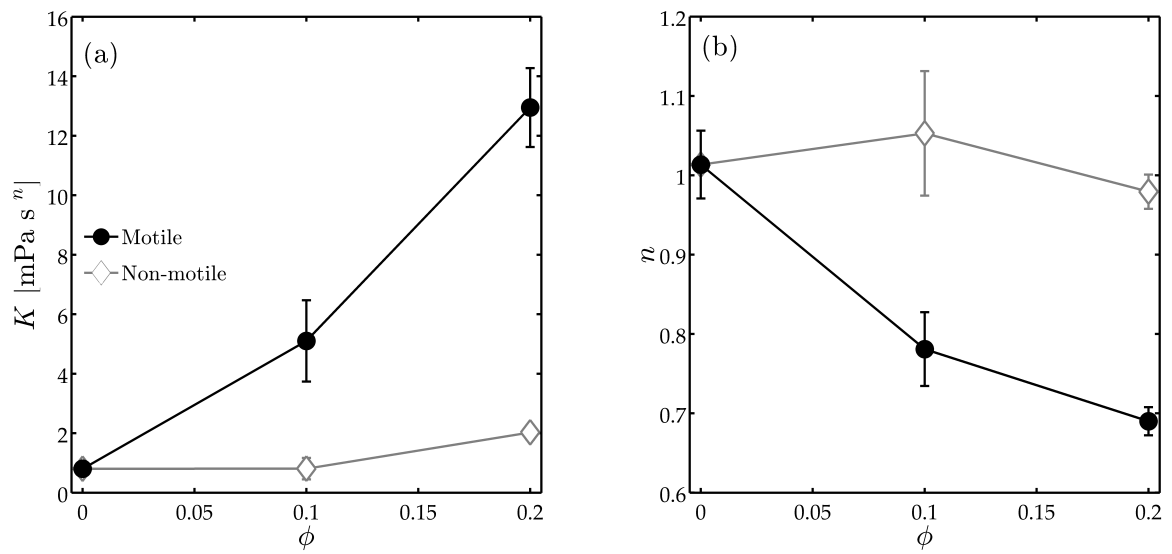

Fig. 8 Estimated consistency (a) and flow index (b) suspensions of motile and non-motile T. chuii cells at different volume fractions. The values were estimated by fitting the data in Figure 7 (a) to the Herschel-Bulkley model (Equation 4). The error bars correspond to one standard deviation. 

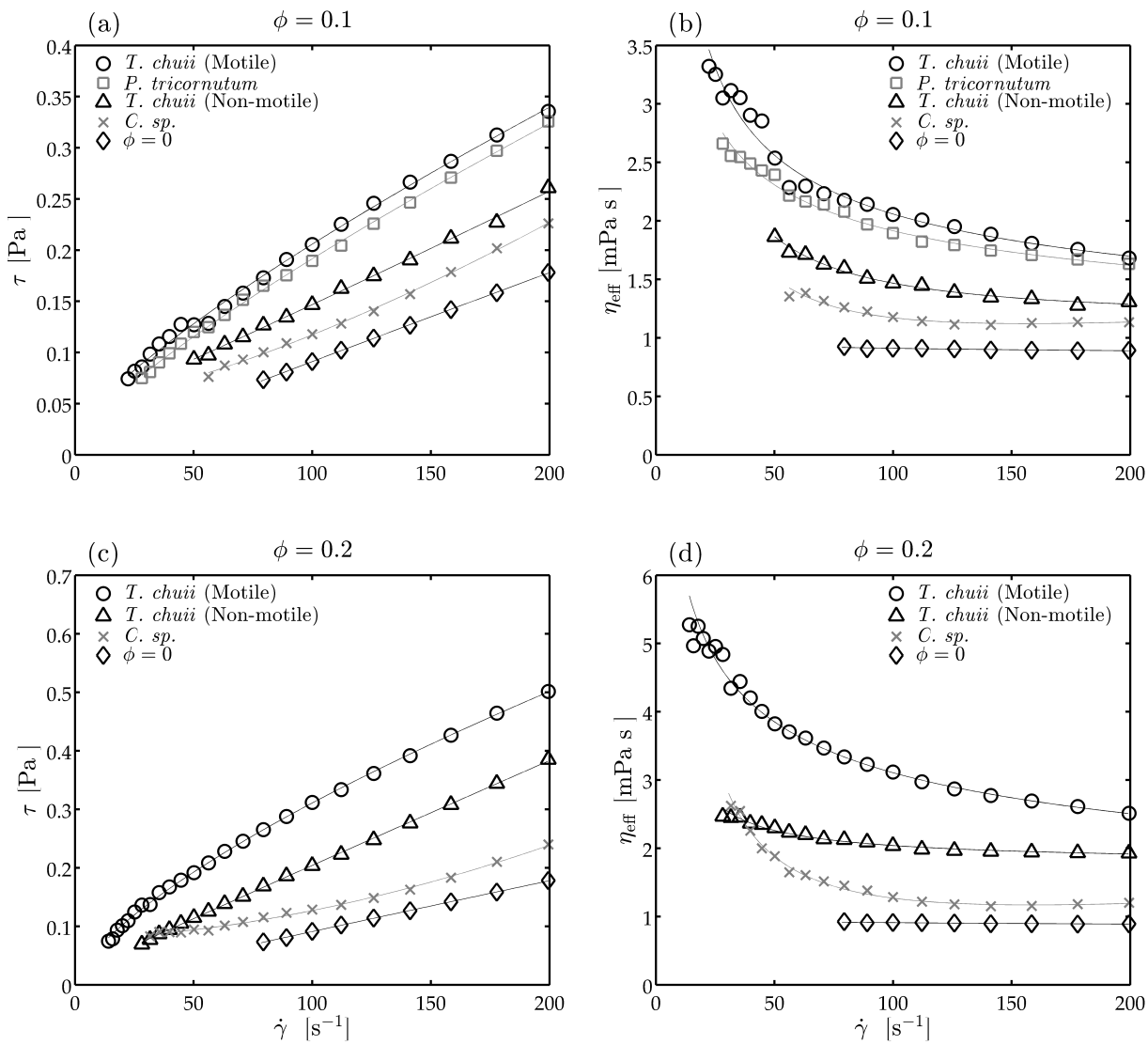

Fig. 9 Variation in the shear stress ( $a$ and c), and effective viscosity (b and d), as a function of strain rate, for suspensions of different algal strains and pure PBS. The data in (a) and (b) were acquired at $\phi=0.1$, while the data in (c) and (d) were acquired at $\phi=0.2$. The lines show the best-fit of the data to the Herschel-Bulkley model (Equation 4). 

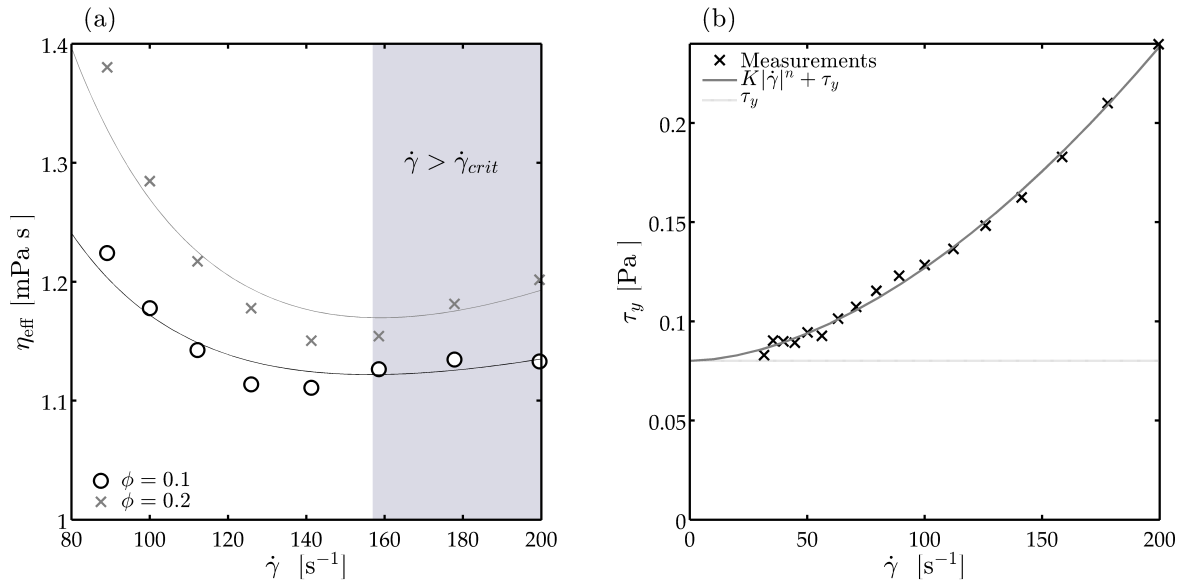

Fig. 10 Variation in the effective viscosity of Chlorella $s p$. suspensions at high shear rates (a) for two cell concentrations. The shaded region $\left(\dot{\gamma}>156 \mathrm{~s}^{-1}\right)$ corresponds to the region where the effective viscosity is predicted to increase with strain rate, according to the Herschel-Bulkley model (Equation 5). The variation in the stress with strain rate for the $\phi=0.2$ case is shown in (b), along with the estimated flow curves (Equation 4) and the contribution of the yield stress. The trend of increasing stress with strain rate (i.e. the shear-thickening behaviour) is particularly clear here.

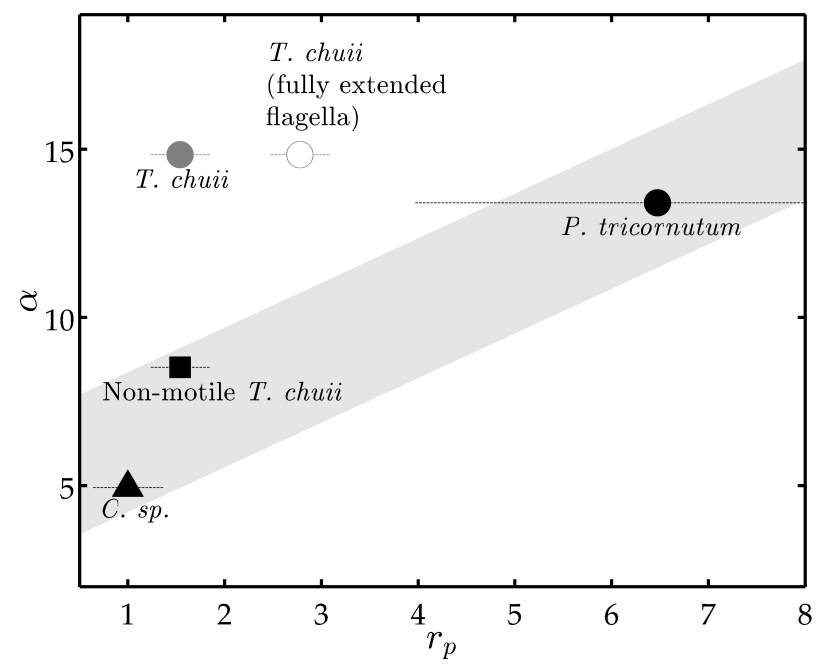

Fig. 11 Intrinsic viscosity, $\alpha=\left(\eta / \eta_{0}-1\right) / \phi$, for each algal suspension as a function of aspect ratio, for $\dot{\gamma}=60 \mathrm{~s}^{-1}$ and $\phi=0.1$. The light grey line indicates a linear-fit to the non-motile data (black symbols). 